\title{
Studies of stainless steel exposed to sandblasting
}

Paweł Horodek,

Marat K. Eseev, Andrey G. Kobets

\begin{abstract}
The influence of sandblasting on surface and subsurface of stainless steel is investigated using variable energy positron beam (VEP), positron annihilation spectroscopy (PAS), scanning electron microscopy (SEM), and atomic force microscopy (AFM). Samples of stainless steel were blasted using $110 \mu \mathrm{m}$ particles of $\mathrm{Al}_{2} \mathrm{O}_{3}$ under different pressure and time duration. In the case of sandblasting for $90 \mathrm{~s}$, the reduction of positron diffusion length depending on the applied pressure was observed. Sandblasting during $30 \mathrm{~s}$ leads only to the reduction of positron diffusion length to about $60 \mathrm{~nm}$ for all samples. Positron lifetimes close to 170 ps measured using positrons emitted directly from the source point to the presence of vacancies on the dislocation lines. SEM and AFM images show that surface roughness depends rather on pressure of sandblasting than time of exposition.
\end{abstract}

Key words: stainless steel $\bullet$ sandblasting $\bullet$ defects $\bullet$ positron beam

\section{P. Horodek ${ }^{\bowtie}$}

Joint Institute for Nuclear Research,

6 Joliot Curie Str., 141980 Dubna, Moscow region, Russian Federation

and Institute of Nuclear Physics of the Polish Academy of Sciences,

152 Radzikowskiego Str., 31-342 Kraków, Poland, E-mail: Pawel.Horodek@ifj.edu.pl

\section{K. Eseev}

Northern Arctic Federal University, 163002 Severnaya Dvina Emb. 17, Arkhangelsk, Russian Federation

\section{A. G. Kobets}

Joint Institute for Nuclear Research,

6 Joliot Curie Str., 141980 Dubna, Moscow region, Russian Federation

and Institute of Electrophysics and Radiation

Technologies NAS of Ukraine,

28 Chernyshevsky Str., 61002 Kharkov, Ukraine

Received: 19 June 2015

Accepted: 31 August 2015

\section{Introduction}

The knowledge on the impact of the given surface treatment process on the tribological properties of the material is important from the practical point of view. Management of the production process, preparing better quality parts of machines and devices, protection from corrosion, and limiting of the wear may need this kind of knowledge.

Sandblasting is a cold working process used to clean surfaces and remove corrosion. The stream of high-speed abrasive particles hits the surface, removes oxides, and forms a clean surface. In this way, in addition next to changes in tribology of the surface, e.g. roughness, the local plastic deformations below it are generated. This causes the increase in the microhardness and residual stresses in a deformed layer.

As a result of plastic deformations, lattice defects appear, and they can be detected using positron annihilation spectroscopy (PAS). PAS is useful in studying changes undetectable by other popular methods such as microhardness test, electron microscopy, or X-ray diffraction. There are numerous works concerning PAS studies on the influence of different surface modification processes such as ion implantation [1], sliding [2], and cutting [3] on the deformed zone properties. Additionally, Dryzek [4] examined pure aluminum and aluminum alloy exposed to sandblasting. In this case, samples were blasted with air pressure 6.5 bar with silicon carbide particles of less than $0.5 \mathrm{~mm}$ in diameter. The exponential decay of defect concentration with in- 
creasing depth from the surface was observed for both materials. The thickness of defected zone was close to $300 \mu \mathrm{m}$. In case of pure aluminum, divacancies were recognized, while in aluminum alloy, vacancy clusters were larger, probably consisted of three vacancies.

Our paper reports the influence of sandblasting with different time of exposition and pressures on the changes in the structure and surface of stainless steel. These are monitored by variable energy positron beam (VEP) and conventional positron annihilation spectroscopy (PAS). Additionally, the surface roughness is examined with scanning electron microscopy (SEM) and atomic force microscopy (AFM).

\section{Experimental details}

The stainless steel (SS) with chemical composition of $0.11 \mathrm{C}, 0.6 \mathrm{Si}, 1.8 \mathrm{Mn}, 10.3 \mathrm{Ni}, 0.01 \mathrm{~S}, 0.03 \mathrm{P}$, $18.6 \mathrm{Cr}, 0.2 \mathrm{Cu}$, and the rest Fe (all in mass \%) was used in this study. The samples were cut into $20 \times$ $10 \times 3 \mathrm{~mm}^{3}$ blocks and polished to get a smooth surface. Then two sets of seven identical samples were annealed in $800^{\circ} \mathrm{C}$ for 2 hours in vacuum conditions of $10^{-8}$ bar and slowly cooled down to room temperature. In this way, samples in the same state containing residual amount of defects were obtained. Next, two specimens were saved as a reference sample (RF) while the rest of them were sandblasted using Renfert Vario Basic Jet blaster. The abrasive material Edelkorund containing 99.8\% of aluminum oxide $\left(\mathrm{Al}_{2} \mathrm{O}_{3}\right)$ with size of $110 \mu \mathrm{m}$ was applied. The surfaces were blasted perpendicularly with the distance of $10 \mathrm{~mm}$ between a sample and a nozzle under the pressure 1, 3, and 5 bar during 30 and $90 \mathrm{~s}$.

VEP is operating as a part of Low Energy Positron Toroidal Accumulator (LEPTA) facility at the Joint Institute for Nuclear Research (JINR) in Dubna [5]. The positrons emitted from ${ }^{22} \mathrm{Na}$ source of $25 \mathrm{mCi}$ activity were moderated onto frozen $\mathrm{Ne}(7 \mathrm{~K})$ under pressure of $10^{-8}$ Torr. Then, they were accelerated to the demanded energy in the range between $50 \mathrm{eV}$ and $30 \mathrm{keV}$ and in the form of a beam of $5 \mathrm{~mm}$ diameter and intensity of $10^{5} \mathrm{e}^{+} / \mathrm{s}$ implanted into the sample. $511 \mathrm{keV}$ quanta from the annihilation process were registered by Doppler broadening (DB) of annihilation gamma line spectrometer with $1.2 \mathrm{keV}$ energy resolution interpolated to $511 \mathrm{keV}$.

From measured lines, the so-called S and $\mathrm{W}$ parameters were extracted. The first one is defined as the ratio of area under the central part of the annihilation line to the total surface area under this line. The second one is calculated as the ratio of the area under the wing part of annihilation line to the whole area under the line. S parameter is strictly correlated with the type of defects and their concentration, i.e. the higher its value, the greater is the concentration of defects. The connection of two parameters can give information about a kind of defects.

Positron lifetimes were measured with a conventional fast-fast spectrometer. It was constructed from two $\mathrm{BaF}_{2}$-based detectors and standard ORTEC electronic units: the time resolution was $250 \mathrm{ps}$. The isotope ${ }^{22} \mathrm{Na}$ with activity $32 \mu \mathrm{Ci}$ enveloped into a $7 \mu \mathrm{m}$ thick Kapton foil was applied. Positron source was placed between two identical samples in front of the scintillator detectors of the positron lifetime spectrometer. Obtained spectra including $10^{6}$ counts were deconvoluted by LT code [6], subtracting the background and the source components.

The surface characterization was performed using scanning electron microscope Tescan Vega3 SBH and atomic force microscope AFM/STM SOLVER Nano (NT-MTD).

\section{PAS measurements}

The dependencies of S parameter on positron implantation energy (bottom axis) and mean implantation depth (top axis) for the reference (RF) and sandblasted samples for 30 and $90 \mathrm{~s}$ are presented in Figs. 1a and $1 \mathrm{~b}$, respectively. The mean implantation depth $\bar{z}$ was estimated using the formula

$$
\bar{z}=\frac{A E^{n}}{\rho}
$$

where $E$ is positron energy in $\mathrm{keV}, A=2.62 \mu \mathrm{g} /$ $\left(\mathrm{cm}^{2} \cdot \mathrm{keV}^{n}\right), n=1.692$ are Makhov's parameters for iron, and $\rho=7.87 \mathrm{~g} / \mathrm{cm}^{3}$ stands for density [8]. In this experiment, investigated depth was between $0.02 \mathrm{~nm}$ and $1.05 \mu \mathrm{m}$ under the surface. In all cases, $\mathrm{S}$ parameter decreases with increasing positron energy and saturates in the region of higher energy. The level of S parameter saturation gives information about the presence of open volume defects. Higher value of S parameter in the saturation area
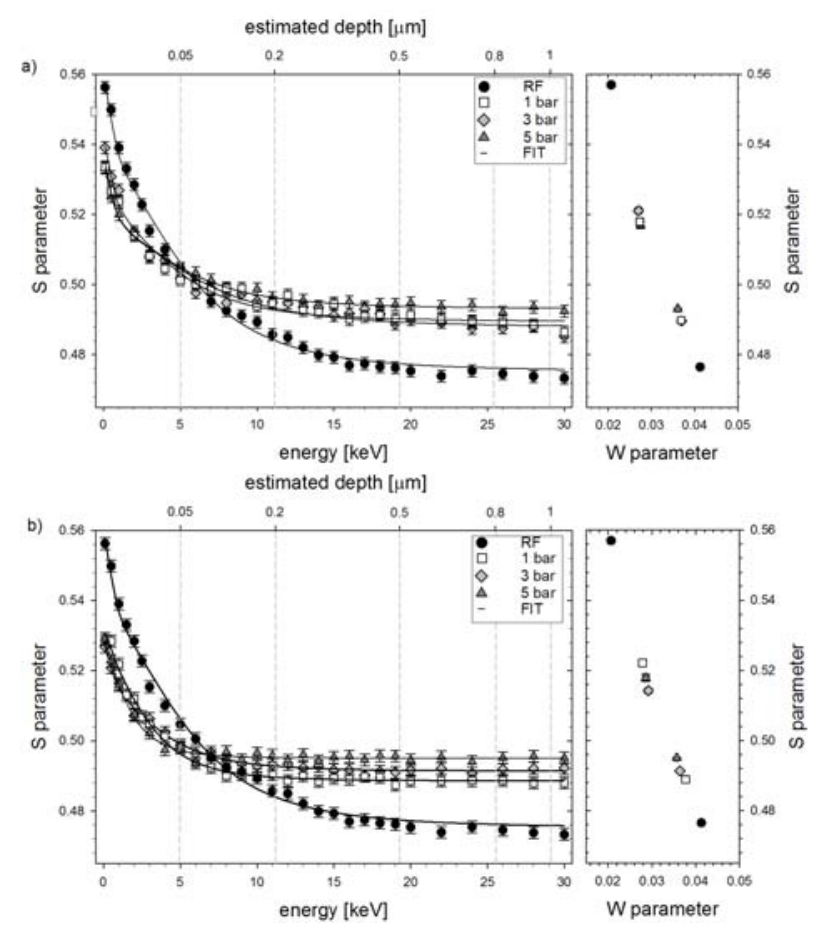

Fig. 1. The dependencies of the $S$ parameter on the incident positron energy (on left) and on $\mathrm{W}$ parameter (on the right) for RF as well as for samples sandblasted for $30 \mathrm{~s}$ (a) and $90 \mathrm{~s}$ (b). Solid black lines represent the best fit using VEPFIT code [7]. 


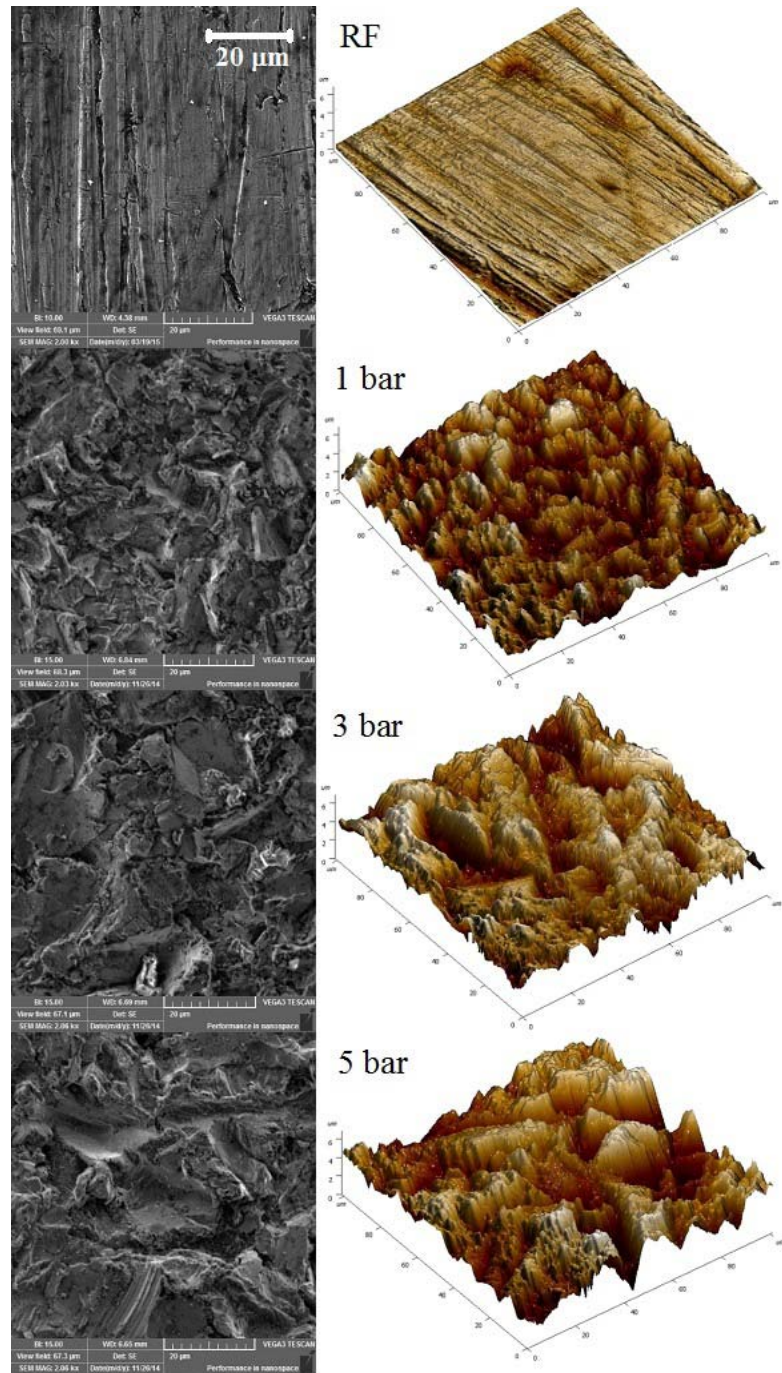

Fig. 2. SEM: BI, 15; WD, $6.84 \mathrm{~mm}$; view field, $68.3 \mathrm{~mm}$; Detector - SE, SEM MAG - $2.03 \mathrm{kx}$ (on the left) and AFM (on the right) images of RF and samples sandblasted for $90 \mathrm{~s}$.

can be attributed to higher defects concentration. The profile representing RF (black circles) as annealed and containing residual defects only is located below the sandblasted ones. The saturation in this case appears in the region of $15 \mathrm{keV}$. For sandblasted samples, faster saturation of $\mathrm{S}$ parameter values is clearly visible. It is observed around $10 \mathrm{keV}$ for sandblasting for $30 \mathrm{~s}$ and close to $5 \mathrm{keV}$ for $90 \mathrm{~s}$. It points to the reduction of positron diffusion lengths $\left(L_{+}\right)$. Additionally, the saturation of profiles occurs for higher values of $S$ parameter in comparison to RF sample. The influence of different pressures used in the process of $90 \mathrm{~s}$ sandblasting is marked stronger than during $30 \mathrm{~s}$ treatment.

More convenient analysis can be performed by fitting $\mathrm{S}$ parameter profiles using VEPFIT program [7]. The fitting procedure was performed by taking into account single layer model and Makhov's parameters $-A, n$ (mentioned above), and $m=1.766$. The best fits are marked with solid black lines and corresponding parameters are presented in Table 1.

Positron diffusion length obtained from VEPFIT program [7] for RF sample equals $79 \mathrm{~nm}$. Wu and Jean [9] reported $L_{+}$of $78 \mathrm{~nm}$ and $59 \mathrm{~nm}$ in the bulk of 304 and 316 steels, respectively. Thus, the obtained values correspond to these reported by other authors. Sandblasting for $30 \mathrm{~s}$ reduces $L_{+}$to ca. $60 \mathrm{~nm}$, which seems to be rather independent on the pressure applied during this treatment. Small change is visible in the values of $S_{\text {bulk }}$ parameters. $\Delta S_{\text {bulk }}$ defined as the difference in $S_{\text {bulk }}$ parameter for sandblasted samples to $S_{\text {bulk }}$ for $\mathrm{RF}$ is equal 0.013 for 1 and 3 bar, while for 5 bar, it arises to 0.016 . Sandblasting for 90 s shows clearly the impact of pressure on the annihilation characteristics. Treatment under the pressure of 1 bar reduces positron diffusion length to $39 \mathrm{~nm}$, while for $5 \mathrm{bar}$, it is equal to only $13 \mathrm{~nm}$. Simultaneously, the above mentioned $\Delta S_{\text {bulk }}$ factor arises from 0.012 for 1 bar to 0.018 for 5 bar. It points to the increase in defect concentration with higher pressure. Additionally, $\Delta \mathrm{S}_{\text {bulk }}$ factors for sandblasting under 1 bar for the time of 30 and $90 \mathrm{~s}$ are comparable.

On the right of Fig. 1, W parameter vs. S parameter for studied samples is shown. Points represent values of these parameters in bulk and surface, but they were obtained by fitting using VEPFIT [7]. For clarity, $\mathrm{W}(E)$ profiles was not presented in this work. In both cases (30 and $90 \mathrm{~s}$ ), all points fall approx. on a straight line. It indicates the domination of the same kind of defects across the scanned adjoined surface layer.

The measurements of the positron lifetime spectra direct on the sandblasted surface revealed only a single component. Table 1 gathered the obtained values for the studied samples. The mean implantation depth of positrons emitted from ${ }^{22} \mathrm{Na}$ into the

Table 1. Parameters characterizing studied samples: $L_{+}$as a positron diffusion length, $\mathrm{S}_{\text {bulk }}$ is the value of $\mathrm{S}$ parameter in saturation and $S_{\text {surf }}$ on the surface obtained from VEPFIT [7], positron lifetimes $\tau$ from LT measurements, $S_{a}$ representing arithmetical mean height, $\mathrm{S}_{p}$ maximum peak height and maximum pit height $\mathrm{S}_{v}$ from AFM

\begin{tabular}{lccccccc}
\hline \multicolumn{1}{c}{ Sample } & $L_{+}[\mathrm{nm}]$ & $\mathrm{S}_{\text {bulk }}$ & $\mathrm{S}_{\text {surf }}$ & $\tau[\mathrm{ps}]$ & $\mathrm{S}_{a}[\mu \mathrm{m}]$ & $\mathrm{S}_{p}[\mu \mathrm{m}]$ & $\mathrm{S}_{v}[\mu \mathrm{m}]$ \\
\hline \multicolumn{1}{c}{$\mathrm{RF}$} & $79(1)$ & $0.477(2)$ & $0.557(1)$ & $111(1)$ & $0.13(2)$ & $0.63(16)$ & $1.36(27)$ \\
\hline \multicolumn{7}{c}{$30 \mathrm{~s}$} \\
1 bar & $63(3)$ & $0.490(1)$ & $0.518(1)$ & $164(1)$ & $0.42(2)$ & $1.86(16)$ & $2.24(22)$ \\
$3 \mathrm{bar}$ & $58(3)$ & $0.490(1)$ & $0.521(1)$ & $170(1)$ & $0.56(7)$ & $2.22(38)$ & $2.54(28)$ \\
5 bar & $64(3)$ & $0.493(1)$ & $0.517(1)$ & $172(1)$ & $0.88(5)$ & $3.03(44)$ & $3.99(4)$ \\
& & & & & & \\
1 bar & $39(1)$ & $0.489(2)$ & $0.522(1)$ & $169(1)$ & $0.44(2)$ & $1.75(80)$ & $2.18(16)$ \\
3 bar & $26(1)$ & $0.491(2)$ & $0.514(1)$ & $173(1)$ & $0.77(7)$ & $2.88(29)$ & $4.73(78)$ \\
5 bar & $13(1)$ & $0.495(2)$ & $0.518(1)$ & $172(1)$ & $0.96(4)$ & $3.25(41)$ & $4.15(41)$ \\
\hline
\end{tabular}


SS amounts to $29 \mu \mathrm{m}$. The positron lifetime for RF equaled 111 ps can be attributed to annihilation in the defect-free lattice. Lifetime values obtained for deformed samples vary between 164 and 173 ps. Because the positron lifetime in the vacancy of the perfect lattice is equal to $180 \mathrm{ps}$, the lower value can point to the annihilation in single vacancies on dislocations edges.

\section{SEM and AFM analysis}

Figure 2 shows SEM (on the left) and AFM (on the right) images of RF and samples sandblasted for $90 \mathrm{~s}$. The RF surface is characterized by oriented in one direction trenches formed during preparation. Sandblasting changes definitely this type of morphology introducing random deformations created as a result of contact of the surface with abrasive particles. In this way, in SEM pictures, irregular squeezes created on the surface are clearly visible. Higher pressure during blasting is the reason of bigger area of the trace. The micrographs obtained from AFM tests show surface occupied by nonregular peaks and valleys. Application of higher pressure of abrasive particles leads to the reduction of sharp peaks against bigger ones and deeper valleys.

The AFM measurements offer the possibility of quantitative analysis of the surface roughness using special parameters. In this way, the mean roughness defines arithmetical mean height $\mathrm{S}_{a}$; additionally, maximum peak height $\mathbf{S}_{p}$ defined as the height between the highest peak and the mean plane, maximum pit height $\mathrm{S}_{v}$ as the depth between the mean plane and the deepest valley. The area $100 \times$ $100 \mu \mathrm{m}^{2}$ was measured five times and results were averaged. Results are presented in Table 1 .

The $\mathrm{S}_{a}$ parameter, that is, the average roughness, for RF equals $0.13 \mu \mathrm{m}$, but in the case of sandblasted samples, it varies from 0.42 to $0.96 \mu \mathrm{m}$ in dependency on the applied pressure. It is important that the small differences between times of sandblasting are visible. When maximal height of peak in the RF sample equals to $0.63 \mu \mathrm{m}$, this parameter becomes three times as big as for sample blasted under 1 bar and five times as high as for 5 bar. The impact of blasting time is imperceptible. Similar situation appears for $S_{v}$ parameter where it arises from $1.36 \mu \mathrm{m}$ for RF to about $2.2 \mu \mathrm{m}$ for 1 bar to ca. $4 \mu \mathrm{m}$ for 5 bar. The time of exposition does not reveal an important impact on this characteristic.

\section{Summary}

The stainless steel exposed to sandblasting was investigated using VEP, PAS, SEM, and AFM methods. Analysis of S parameter profiles points to the reduc- tion of positron diffusion lengths attributed to the creation of lattice open volume defects. In the case of blasting for $90 \mathrm{~s}$, increasing pressure is reflected in decreasing positron diffusion length. For $30 \mathrm{~s}$, only shortening of diffusion length was observed without the influence of pressure. Positron lifetimes measured on the sandblasted surfaces indicate the presence of vacancies on the edge of dislocations. The SEM and AFM images show the deformation of sandblasted surfaces. The surface roughness seems to be more sensitive to the pressure applied during the process than the time of exposition.

Acknowledgments. Part of works was supported by the Ministry of Education and Science of the Russian Federation within the project no. 3635 .

\section{References}

1. Hu, Y., Xue, X., \& Wu, Y. (2014). Slow positron beam study of hydrogen ion implanted $\mathrm{ZnO}$ thin films. Radiat. Phys. Chem., 101, 20-23. DOI: 10.1016/j. radphyschem.2014.03.045.

2. Dryzek, J. (2011). Positron studies of subsurface zone in pure iron induced by sliding. Tribol. Lett., 42, 9-15. DOI: 10.1007/s11249-010-9742-x.

3. Horodek, P., Dryzek, J., \& Wróbel, M. (2012). Positron annihilation study of defects induced by various cutting methods in stainless steel grade 304. Tribol. Lett., 45, 341-347. DOI: 10.1007/ s11249-011-9890-7.

4. Dryzek, E. (2003). Defect depth profiling after sphere indentation and blasting in aluminium and aluminium alloy detected by positron annihilation. J. Mater. Sci., 38, 3755-3763. DOI: 10.1023/A:1025976031584.

5. Sidorin, A., Meshkov, I., Akhmanova, E., Eseev, M., Kobets, A., Lokhmatov, V., Pavlov, V., Rudakov, A., \& Yakovenko, S. (2013). The LEPTA facility for fundamental studies of positronium physics and positron spectroscopy. Mater. Sci. Forum, 733, 291-296. DOI: 10.4028/www.scientific.net/MSF.733.291.

6. Kansy, J. (1996). Microcomputer program for analysis of positron annihilation lifetime spectra. Nucl. Instrum. Methods Phys. Res. Sect. A-Accel. Spectrom. Dect. Assoc. Equip., 374, 235-244. DOI: 10.1016/0168-9002(96)00075-7.

7. Van Veen, A., Schut, H., Clement, M., Kruseman, A., Ijpma, M. R., \& De Nijs, J. M. M. (1995). VEPFIT applied to depth profiling problems. Appl. Surf. Sci., 85, 216-224. DOI: 10.1016/0169-4332(94)00334-3.

8. Dryzek, J., \& Horodek, P. (2008). GEANT4 simulation of slow positron beam implantation profiles. Nucl. Instrum. Methods Phys. Res. Sect. B-Beam Interact. Mater. Atoms, 266(18), 4000-4009. DOI: 10.1016/j.nimb.2008.06.033.

9. Wu, Y. C., \& Jean, Y. C. (2007). Hydrogen-damaged defects near the surface in heavily deformed iron and steels investigated by slow positron annihilation spectroscopy. Phys. Status Solidi C, 4, 3506-3509. DOI: 10.1002/pssc.200675826. 